\title{
Can A Community-Based Weight Management Programme, that is Scalable, Provide Effective Diabetes Management Support for Adults with T1D or T2D: A Mixed Methods Evaluation
}

\author{
Amanda Avery*1, Jenny Barber ${ }^{2}$, Asma Zina Belheddad ${ }^{3}$, Carolyn Pallister ${ }^{2}$, Josef Toon $^{4}$, Sarah Bennett ${ }^{4}$ and Jacquie \\ Lavin $^{4}$ \\ ${ }^{1}$ Associate professor in Nutrition \& Dietetics, Division of Food, Nutrition \& Dietetics, School of Biosciences, University of Nottingham, UK \\ ${ }^{2}$ Nutrition \& Health, Slimming World, UK \\ ${ }^{3}$ Resident Doctor in Endocrinology, Algeria \\ ${ }^{4}$ Research \& Scientific Affairs, Slimming World, UK \\ *Corresponding author: Dr. Amanda Avery, Associate professor in Nutrition \& Dietetics, Division of Food, Nutrition \& Dietetics, School \\ of Biosciences, University of Nottingham, UK
}

\section{Research in Context Summary}

What is already known about the subject?

a) Weight management may prevent, help manage and reverse T2D and improve glycaemic control and metabolic risk, without the need to increase insulin doses, in those with T1D affected by overweight or obesity.

b) Weight loss is the primary driver of T2D remission and that it does not matter what dietary approach is used providing the person is able to follow the diet in order to achieve long-term weight loss.

c) Given the increasing prevalence of T2D and T1D that is associated with overweight or obesity, scalable solutions are required to support people with either type of diabetes to better manage their weight

What is the Key Question?

Can a community-based weight management programme, that is scalable and where people can either be referred to or selfselect to join, offer weight management support to adults with T2 and T1D in order to improve their glycaemic control and, for some with T2D, lead to the reversal of their diabetes management. Can this support lead to better mental well-being and be easy to follow in order to achieve long-term improvements?

What are the new findings?

a) $62.3 \%$ study participants, members of Slimming World, achieved a weight loss $\geq 10 \%$ and were 7.3 times more likely to achieve a HbA1c level of $\leq 48 \mathrm{mmol} / \mathrm{mol}$ compared to those achieving smaller weight losses (OD: 7.261, 95\%CI= 2.583-20.409). Those study participants who were referred by a healthcare professional did better.

b) $33.9 \%$ study participants with T2D suggested their diabetes diagnosis was reversed following the lifestyle changes made through membership at Slimming World. This number increased to over $50 \%$ in those achieving $\geq 10 \%$ weight loss.

c) The majority of study participants found it very easy or easy to follow the lifestyle guidance encouraged by Slimming World. At the time of the survey, mental health well-being had improved.

How might this impact clinical practice?

Scalable options are required to support people with T1 \& T2D to better manage their weight and for those with T2D to promote a significant weight loss that may help reverse their condition. Less intensive, community-based options that are accessible to all people for both when and for how long they require the support need to be considered. Referral and support from a healthcare professional may improve the outcomes 


\section{Abstract}

Aim/Hypothesis: This mixed-methods evaluation investigates the impact of Slimming World (SW) membership on weight, glycaemic control and mental-health related quality of life in adults with both T1D and T2D who either self-referred or were referred by a healthcare professional. It also evaluates the ease of application and the accessibility of the support offered to determine longterm sustainability of the lifestyle changes made. The hypothesis was that both glycaemic control and improvements in mental well-being are positively associated with weight loss and that participants would find the intervention easy to follow and achieve weight loss $(\geq 10 \%)$.

Methods: Adult members of SW with diabetes (T1 \& T2D) were invited to complete a questionnaire available on SW's website for a week, with qualitative and quantitative questions including age-range, length of membership, self-reported weight, height and $\mathrm{HbA1c}$ levels before and at the time of survey ( $\mathrm{t} 1$ ), medication use, questions related to changes in mental well-being and their experience of being able to integrate diet and physical activity advice provided by their healthcare team. Statistical analyses were undertaken on SPSS Statistics (v. 26).

Results: Among 268 respondents, 83.2\% had T2D. 88.7\% (238) were female and median age of surveyed members was 50-59 years. Median length of membership, 1-2years; median time since diagnosis, 6-10 years and 68.5\% reported being diagnosed $\geq 6$ yrs. Where data was provided, mean (SD) BMI and HbA1c on joining were $38.9 \pm 7.83 \mathrm{~kg} / \mathrm{m}^{2}$ and $76.6 \pm 37.4 \mathrm{mmol} / \mathrm{mol}$ with $74.4 \%$ reporting a HbA1c above $53 \mathrm{mmol} / \mathrm{mol}$. Reported mean \% weight and HbA1c reductions since joining were 13.0 $\pm 9.03 \%$ and $21.9 \pm 29.7 \mathrm{mmol} / \mathrm{mol}$ respectively at $\mathrm{t} 1.62 .3 \%$ achieved a weight loss $\geq 10 \%$ and were 7.3 times more likely to achieve a HbA1c level $\leq 48 \mathrm{mmol} / \mathrm{mol}$ compared to those achieving smaller weight losses (OD: $7.261,95 \% \mathrm{CI}=2.583-20.409$ ). Reduction in HbA1c levels was independent of diabetes duration or insulin therapy status. A third (33.9\%) of respondents with T2D suggested their diabetes diagnosis had been reversed. Those referred for weight loss by their healthcare professional had greater HbA1c reductions $(\mathrm{p}<0.05)$. At $1,44.0 \%$ respondents (T2D) indicated their diabetes medication had been reduced or stopped. This increased to $60.8 \%$ where weight loss $\geq 10 \%$. At $\mathrm{t} 1,80.4 \%$ showed improved mental-health related quality of life $(\mathrm{p}<0.05) .75 .6 \%$ of respondents (T1D $68.4 \%$; T2D 76.5\%) reported finding it very easy/easy to incorporate personal lifestyle advice from their healthcare practitioner into the healthy eating and activity plan. Very few $(n=20)$ reported challenges.

Conclusions: Slimming World support is a feasible and effective strategy in reducing weight and improving glycaemic control in adults with either T1 or T2D, with almost two-thirds of the study population achieving $\geq 10 \%$ weight loss and being able to reduce their diabetes medications and/or reverse their diabetes diagnosis. Mental-health related quality of life can also be improved. Referral by healthcare professionals increased the improved glycaemic control achieved and where people had been given advice by their healthcare professional, it was generally easy to incorporate this guidance.

\section{Introduction}

Diabetes is associated with overweight or obesity in $90 \%$ of cases with type 2 diabetes, (T2D), and a growing proportion of those with type 1 diabetes (T1D). Weight management has been shown to prevent, help manage and reverse T2D, and improve glycaemic control and metabolic risk, without the need to increase insulin doses, in those with T1D affected by overweight or obesity.

Previously, a very low-calorie diet has been reported to result in a reversal of $\beta$ cell failure and a significant decrease in insulin resistance in participants with T2D [1]. A systematic review suggested that a number of dietary approaches can be effective in decreasing body weight, achieving better glycaemic control and reducing cardiovascular risk in T2D, including Mediterranean, low carbohydrate, low glycaemic index and high protein diets [2]. People without T2D at baseline and with a BMI $\geq 30 \mathrm{~kg} / \mathrm{m}^{2}$ who underwent bariatric surgery had a significantly reduced incidence of T2D after seven years of follow up compared with controls (hazard ratio: $0.20, p<0.0001$ ] [3]. A further systematic review found that a variety of commercial weight loss programmes produce a positive effect on glycaemic control in people with or at risk of T2D [4]. Most recently, weight loss has been proposed to be the primary driver of T2D remission and that it does not matter what dietary approach is used providing the person is able to follow the diet in order to achieve long-term weight loss [5].

The prevalence of obesity in people with T1D is increasing, driven by the increase in its prevalence in the general population and the intensive therapeutic approaches to the management of hyperglycaemia in T1D [6]. Obesity leads to insulin-resistance leading to the increase in insulin requirement and more weight gain. Compared with T2D, there is a scarcity of studies examining the effect of weight management interventions in T1D. Weight reduction cannot prevent the occurrence of T1D but current data show that it facilitates its management and reduces associated metabolic risk in patients with overweight or obesity [7]. Weight loss following bariatric surgery in people with T1D can result in reduced insulin requirement and improved glycaemic control with a pooled post-operative HbA1c change of $0.5 \%(p=0.01)$ [8]. In addition to its association with diabetes, excess body fat excess is an independent risk factor of all-cause mortality and morbidity including mental health issues [9]. There is a bi-directional link between diabetes and depression [10]. Consequently, addressing overweight and obesity, independently from the direct impact on diabetes management, further contributes to decreasing these 
wider health risks. Given the increasing prevalence of T2D and T1D that is associated with overweight or obesity, scalable solutions are required to support people with either type of diabetes to better manage their weight.

Slimming World (SW) is a community-based, non-surgical weight management programme that people with or without diabetes can access in the UK and Ireland (group support) or more globally (online support). SW supports its members through weekly group sessions and an online platform with a multi-component approach that focuses on psychological as well as physiological aspects of weight control to encourage the formation of new healthier eating habits and increases in physical activity levels. The underpinning psychological approach is built on a number of behaviour change techniques including self-determination theory and the commitment model, but essentially the programme offers positive support and reinforcement, with no criticism or judgement. SW's dietary approach focuses on satiety advocating ad libitum intake of many low energy dense foods that are nutrient rich with controlled amounts of higher energy dense, nutrient poor foods. Previous studies have found that members of SW with diabetes significantly reduce their weight, improve their HbA1c levels and that improvements are correlated to weight loss $[11,12]$, but there has been no consideration as to how people with diabetes find the support offered by SW and how the support complements the advice received from their healthcare team.

This mixed-methods study aims to evaluate the impact of SW on weight, glycaemic control and mental- health related quality of life in adults with both T1D and T2D, and also to evaluate the ease of application and the accessibility of the support offered by SW to determine the long-term sustainability of the lifestyle changes made. It is the first evaluation of a community-based weight management programme to determine whether this level of support can lead to the reversal of a T2D diagnosis in a group of adults who had either chosen to access the support themselves or had been referred by their healthcare team.

\section{Materials and Methods}

\section{Subjects}

Study participants were adults with diabetes who are members of Slimming World (SW) with access to the member website and had been members for any length of duration. Participants joined SW through self-referral or referral by their healthcare professionals and could be group or online members.

\section{Data Collection}

SW members were invited to take part in the survey via the member website. The link to an online survey hosted on Qualtrics was advertised on the SW member website for a week in May 2020. Members who self-selected as living with diabetes upon enrolment in SW were also invited by e-mail to complete the questionnaire. Participants were asked to provide information about their demographics (gender, age category), diabetes condition (type, duration, associated medications), associated medical conditions, the length of time they had been a member of SW, the advice they received from their primary care practitioner regarding diet and physical activity and their experience of being able to combine advice provided by their healthcare team with the support accessed via SW. They were also requested to self-report their weight, height and HbA1c levels before and after joining SW (optional), to indicate whether their T2D was in remission/been reversed, self-report changes in physical activity levels, and provide details on overall mental health-related quality of life. Participants were provided the opportunity to include free text to support their responses to a number of the questions.

\section{Data Analysis}

Descriptive statistics were used to present demographic data. BMI values were calculated from self-reported weight $(\mathrm{kg})$ and height $(\mathrm{m})$ data (weight/height2). Data analysis was undertaken on IBM SPSS Statistics version 26 software. Changes in weight, BMI and $\mathrm{HbA} 1 \mathrm{c}$ were calculated for participants who reported these variables before and after joining SW. NICE guidance for diabetes management recommends a target of $\mathrm{HbA1c}$ below $53 \mathrm{mmol} /$ mol $(7.0 \%)$ as this can reduce the risk of developing micro- and macrovascular complications and below $48 \mathrm{mmol} / \mathrm{mol}$ (6.5\%) signifies a prediabetes state. Thus $53 \mathrm{mmol} / \mathrm{mol}(7.0 \%)$ was used as a standard target as part of the evaluation process and a value below $48 \mathrm{mmol} / \mathrm{mol}(6.5 \%)$ to indicate if the diabetes diagnosis had been reversed. Participants who stated that their diabetes was diagnosed after joining SW were excluded from HbA1c-related analyses. Mean changes $( \pm \mathrm{SD})$ in weight, BMI and HbA1c levels were calculated using paired t-tests. Associations between HbA1c and weight changes were assessed using Pearson correlation. For other variables, independent t-test was used to compare HbA1c changes in two groups while ANOVA plus posthoc analysis were used when comparing three groups or more. A p-value of $\leq 0.05$ was considered statistically significant. For free-text questions, responses to the question related to the ease of combining advice provided by the healthcare team with that provided by SW, content analysis was undertaken using manual coding to convert qualitative data into themes.

A mental health-related quality of life score was created by adapting items from the SF-36 to assess how often participants felt calm and peaceful, (item 1), had a lot of energy (item 2), felt downhearted and low (item 3) and had been a happy person (item 4), with three additional questions assessing how often; participants had been in a sociable mood (item 5), felt stressed (item 6) and felt anxious (item 7). All items were scored from 6 ("All of the time") to 1 ("None of the time"). A score from the scale was calculated by summing scores from all items after reversing questions on negative affect (items 3,6 and 7) to create a total mental health-related quality of life score $(\min =7, \max =42)$ where a higher score indicated a more positive well-being. The scale assessing mental health related quality of life was tested for reliability in multiple ways. Average item-total correlation was high $(\mathrm{r}=0.77)$ and split-half reliability 
showed a high internal consistency estimate ( $\mathrm{r}=0.84)$. Cronbach's alpha analysis also showed the well-being scale had high internal consistency ( $\alpha=0.88$ ) with no items identified as improving the scale if removed with high inter-item correlation $(r=0.52)$.

\section{Results}

\section{Respondent Characteristics}

Of the 268 respondents, $83.2 \%$ had T2D. $88.7 \%$ (238) total respondents were female, and the median age category of surveyed members was 50-59 years with a range 20-29 to >70years (Figure 1). At the time of the survey respondents had, on average, been members of SW for 1-2 years. Those with T1D had a lower median length of membership of 6 months to 1 year. However, some respondents had accessed support for much longer (T1D: 18.2\% 3-5 yrs, $11.4 \%$ > 5yrs; T2D: 23.0\% 3-5yrs, 18.7\% >5yrs). $94.2 \%$ were SW group members (usually attending communitybased group sessions, running as virtual Zoom group sessions at the time of survey due to Covid-19 lockdown restrictions) and 5.8\% were accessing SW's online-only service. All respondents with T1D had been diagnosed before joining SW as had $86.7 \%$ of those with T2D. The median length of time since being diagnosed with diabetes was 6-10 years and $68.5 \%$ reported being diagnosed with diabetes 6 or more years ago. This did differ by diabetes type with $91.3 \%$ of those with T1D being diagnosed 6 or more years ago compared with $63.5 \%$ of those with T2D. The majority $(79.1 \%)$ of those with T2D reported taking some form of diabetes medication (not including insulin) and $16.6 \%$ took insulin.

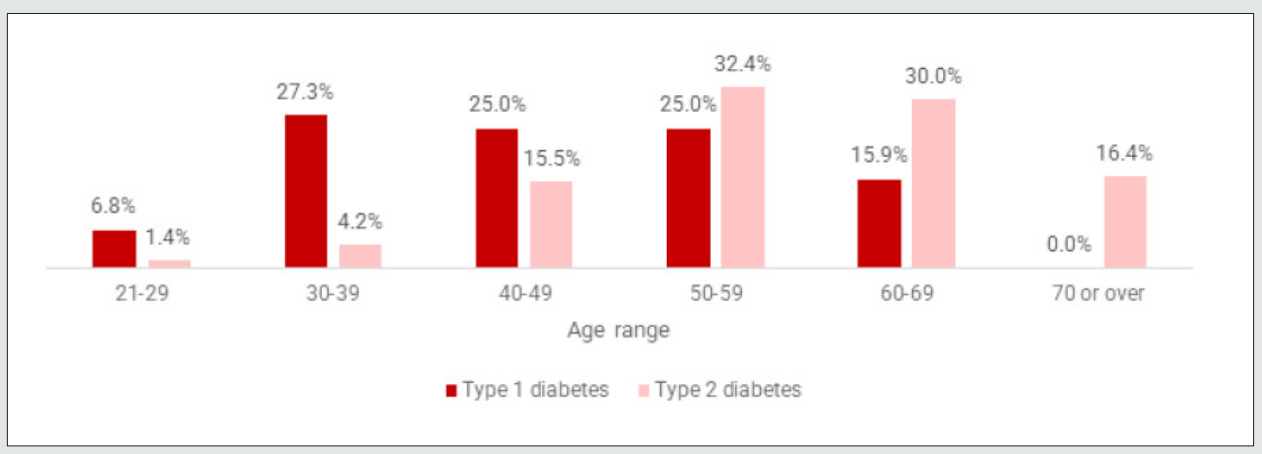

Figure 1:

\section{Outcomes}

\section{Weight Change}

Details of weight on joining SW and at the time of survey was available for $88.1 \%(n=236)$ of the sample. Mean (SD) weight on joining was $106.9 \pm 24.96 \mathrm{~kg}$ and BMI $38.9 \pm 7.83 \mathrm{~kg} / \mathrm{m}^{2}$ (T2D,39.7 \pm 7.83 ; T1D,35.3 \pm 6.80 ). At the time of survey mean weight was $92.7 \pm 22.79 \mathrm{~kg}$ and BMI $33.8 \pm 7.53 \mathrm{~kg} / \mathrm{m}^{2}$, reflecting a reduction in body weight of $13.0 \%$ or $14.2 \mathrm{~kg}(\mathrm{p}<0.001)$ and a BMI reduction of $5.1 \mathrm{~kg} / \mathrm{m}^{2}(\mathrm{p}<0.001)$. Both those with T1D and T2D showed significant weight losses $(\mathrm{p}<0.001)$ with neither group benefitting more than the other (Table 1). Seven respondents (3.6\%), all with T2D, gained weight, one with T1D and four respondents with T2D maintained weight, the remaining all achieving weight loss. 79 (33.5\%) respondents reported a weight change of $\geq 15 \mathrm{~kg}$ (67 T2D; 12 T1D) Overall, $62.3 \%$ of respondents $(n=147)$ had achieved a $10 \%$ weight loss or greater at the time of the survey. $16.5 \%$ were classed as SW target members (had achieved their personally selected weight loss goal and were now maintaining their weight loss).

Table 1: mean (SD) changes and differences in weight and BMI for respondents with T1D or T2D.

\begin{tabular}{|c|c|c|c|c|}
\hline & All Participants & T1D & T2D & Level of significance: (T1D vs T2D) \\
\hline Weight change (\%) & $-13.0 \pm 9.0$ & $-12.5 \pm 7.0$ & $-13.1 \pm 9.4$ & 0.232 \\
\hline Weight change (kg) & $-14.2 \pm 11.2$ & $-12.4 \pm 8.1$ & $-14.6 \pm 11.7$ & 0.217 \\
\hline BMI change $\left(\mathrm{kg} / \mathrm{m}^{2}\right)$ & $-5.1 \pm 3.9$ & $-4.5 \pm 2.9$ & $-5.2 \pm 4.1$ & 0.255 \\
\hline
\end{tabular}

\section{Reported Changes in Blood Glucose Management}

A large proportion of respondents self-reported improvements in blood glucose management (62.6\% of those with $\mathrm{T} 2$ and $75.0 \%$ of those with T1D). For those who had lost at least $10 \%$ of their body weight by the time of the survey, $81.9 \%$ reported improved blood glucose management $(87.5 \%$ of those with type 1 and $80.8 \%$ of those with type 2 diabetes).

\section{HbA1c Data}

94 respondents (35.1\% of the sample) were able to provide data on glycated haemoglobin (HbA1c) for both before joining SW and at the time of the survey. Mean HbA1c before joining SW was $76.6 \pm 37.4 \mathrm{mmol} / \mathrm{mol}(9.2 \pm 5.6 \%)$ and $74.4 \%(\mathrm{n}=70)$ reported a HbA1c above $53 \mathrm{mmol} / \mathrm{mol}(7.0 \%)$. At the time of the survey, $85.1 \%$ respondents reported a reduction in $\mathrm{HbA} 1 \mathrm{c}$ levels 
with mean $\mathrm{HbA} 1 \mathrm{c}$ reducing to $54.7 \pm 23.1 \mathrm{mmol} / \mathrm{mol}(7.2 \pm 4.3 \%)$ $(\mathrm{p}<0.001)$. For those respondents losing $10 \%$ of their baseline body weight, reductions in HbA1c were greater (Table 2). Data analysis showed a positive correlation between reductions in $\mathrm{HbA1c}$ levels and weight loss both in percentage (\%) $(r=0.306 ; p=0.003)$ and absolute difference $(\mathrm{kg})(\mathrm{r}=0.323 ; \mathrm{p}=0.002)$.

Table 2: mean (SD) levels of HbA1c and changes reported for respondents with T1D or T2D.

\begin{tabular}{|c|c|c|c|c|}
\hline & All Participants & T1D & T2D & Level of significance: (T1D vs T2D) \\
\hline HbA1c before joining Slimming World & $76.6 \pm 37.4$ & $71.5 \pm 18.5$ & $78.2 \pm 41.5$ & \\
mmol/mol (\%) & $(9.2 \pm 5.6)$ & $(8.7 \pm 4.0)$ & $(9.3 \pm 5.9)$ \\
$(\mathrm{n}=22)$ & $(\mathrm{n}=72)$ & \\
\hline HbA1c at point of survey & $54.7 \pm 23.1$ & $59.6 \pm 14.3$ & $53.3 \pm 25.0$ & \\
mmol/mol (\%) & $(7.2 \pm 4.3)$ & $(7.6 \pm 3.5)$ & $(7.0 \pm 4.4)$ & \\
\hline HbA1c change & $-21.9 \pm 29.7$ & $-11.9 \pm 14.5$ & $-24.9 \pm 32.4$ & \\
\hline $\begin{array}{c}\text { mmol/mol (\%) } \\
\text { Respondents losing } \geq 10 \% \text { body } \\
\text { weight; HbA1c change }\end{array}$ & $(-2.0)$ & $(-1.1)$ & $(-2.3)$ & \\
\hline mmol/ & $-27.0 \pm 31.2$ & $-16.6 \pm 16.0$ & $-29.2 \pm 33.2$ & \\
$(\mathrm{n}=68)$ & $(\mathrm{n}=12)$ & $(\mathrm{n}=56)$ & 0.079 \\
\hline
\end{tabular}

22.3\% (n=21) respondents reported a HbA1c level below $53 \mathrm{mmol} / \mathrm{mol}$ (7.0\%) before joining SW, whereas 67\% $(\mathrm{n}=63)$ reported achieving this level at the time of the survey. Just $8.5 \%$ $(\mathrm{n}=8)$ reported HbA1c levels below $48 \mathrm{mmol} / \mathrm{mol}(6.5 \%)$ before joining compared with $53.2 \%(n=50)$ at the time of the survey (Figure 2).

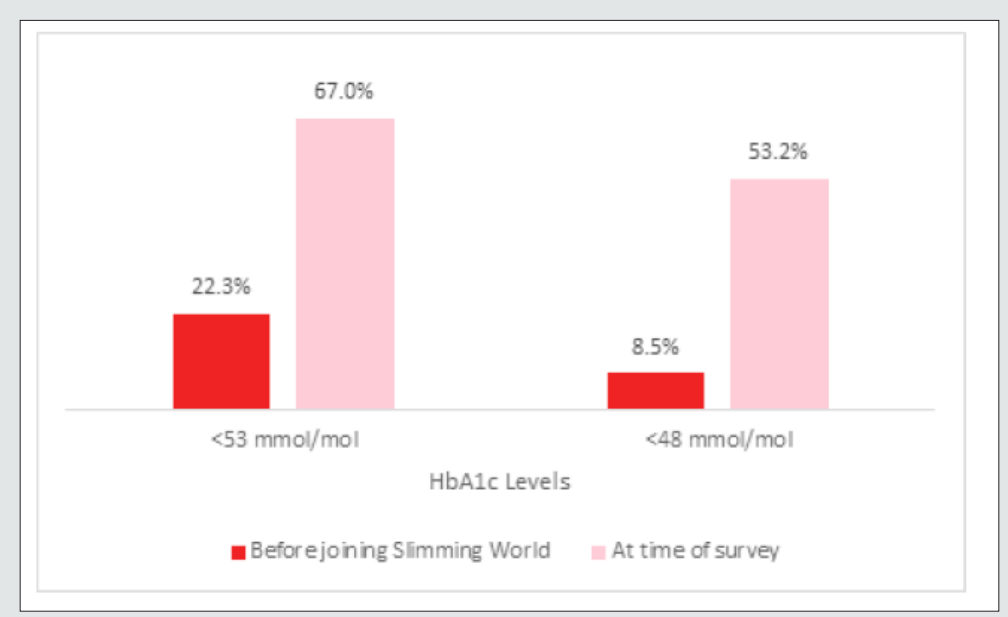

Figure 2: respondents reporting a HbA1c below 48 or $53 \mathrm{mmol} / \mathrm{mol}(6.5$ or $7.0 \%)$ before joining SW compared to at the time of the survey.

For those respondents who achieved a weight reduction of $\geq 10 \%$, they were 7.3 times more likely to achieve a HbA1c level of $48 \mathrm{mmol} / \mathrm{mol}(6.5 \%)$ or less compared to those achieving smaller weight losses (OD: 7.261, 95\%CI= 2.583-20.409). Length of time since diagnosis and reported diabetes duration did not significantly affect changes in HbA1c (5 years and 10 years cut-offs).

\section{Reversal of Type 2 Diabetes}

Since joining Slimming World, $33.9 \%$ of respondents with T2D indicated that they had reversed their condition. Of those who had lost $10 \%$ of their body weight this increased to $50.8 \%$.

\section{Changes in Medication}

Overall, $44.0 \%$ of respondents with T2D indicated that their healthcare team had reduced or stopped their diabetes medication because of them losing weight. In those who had lost $\geq 10 \%$ of their body weight, this increased to $60.8 \%$. Other reported changes in medication included stopping or reducing cholesterol medication $(15.3 \%)$ and blood pressure medication (15.2\%). The free-text supporting information indicated how happy the respondents were to have been able to reduce their medication, particularly insulin. One respondent, representative of a number of similar comments, included the following free-text, 'Before I lost weight, I used $84 \mathrm{U}$ 
insulin per day. I no longer use insulin having lost one third of my starting weight'.

\section{Management of Hypoglycaemia}

99 respondents did indicate that they have experienced hypos but $57.6 \%(n=57)$ saying once per month or less and $25.2 \%(n=25)$ suggesting once per week or more frequently. When asked as to how easy these respondents found managing their hypo with the Food Optimising programme, 36 said either quite or very easy and 38 said quite or very difficult. The remaining respondents indicated that the management was neither easy nor difficult and in terms of strategies to manage hypos, one free-text quote suggested 'asking my GP to reduce my medication, eliminated hypos'.

\section{Physical Activity}

Most respondents (78.6\%, 85.0\% T1D v. 77.3\% T2D; p>0.05) reported increasing their physical activity levels since joining SW.

\section{Changes in other Health Conditions}

Respondents reported benefits in terms of a number of other health conditions since joining SW. For example, of those reporting high cholesterol $(\mathrm{n}=94), 48(51.1 \%)$ reported an improvement at the time of survey. Other improvements included reduced breathlessness $(50.0 \%, 32 / 64)$, blood pressure $(47.2 \%, 52 / 110)$, joint pain $(41.8 \%, 43 / 103)$ and anxiety $(38.0 \%, 35 / 92)$.

\section{Mental-Health Related Quality of Life}

$80.4 \%$ showed improved mental- health related quality of life since joining SW. The mean mental-health related QOL score was $24.0 \pm 6.76$ on joining and this increased to $29.7 \pm 3.89$ at the time of the survey.

The improved quality of life was significantly associated with weight loss (Figure 3).

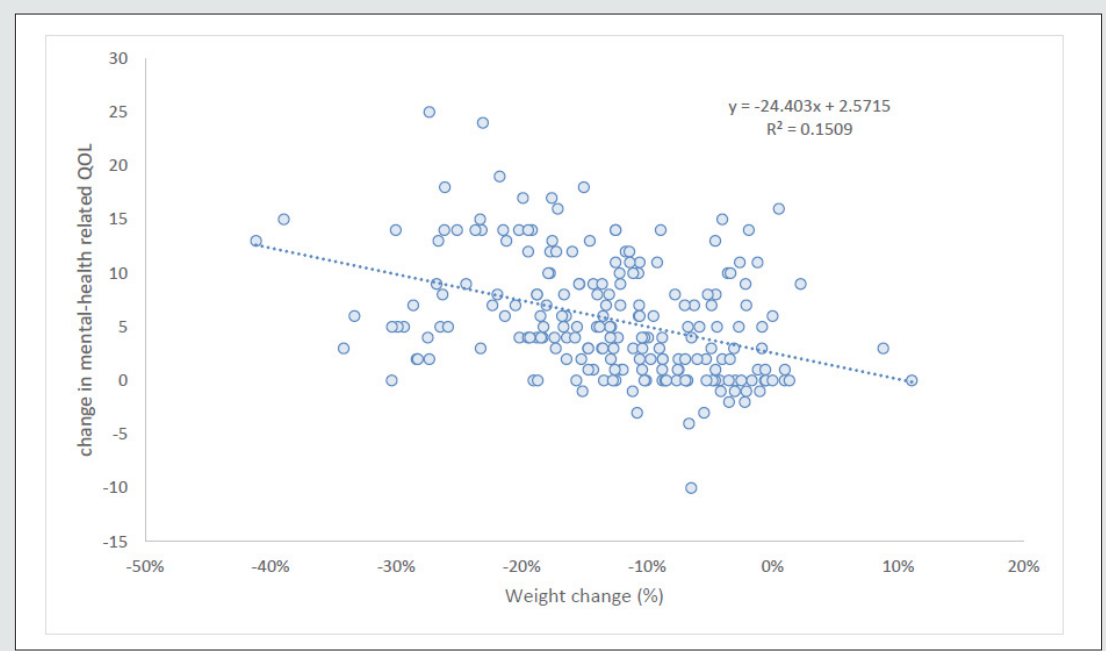

Figure 3: The improved quality of life was significantly associated with weight loss.

\section{Communication with Healthcare Team}

\section{Weight Loss and Health Advice}

The majority (84.0\%) of respondents with T2D reported that their healthcare team had advised them to lose weight to help with their diabetes management, compared to $34 \%$ of those with T1D. Whilst the majority reported their healthcare team were pleased with their weight loss progress (92.0\%), only $27.7 \%$ had been recommended to attend a slimming club or lifestyle change programme. Of those who were recommended to attend a weight management programme by the healthcare team, $47.9 \%(n=34)$ had been referred to SW and these members reported a greater reduction in their HbA1c levels compared with those who reported not receiving such referral, with a mean between-group difference of $-12.3 \mathrm{mmol} / \mathrm{mol}(3.3 \%)$ ( $\mathrm{p}=0.037)$. Most $(92.0 \%)$ reported their healthcare team were pleased with their weight loss progress.

\section{Suitability of Dietary Approach}

Of the 218 respondents who had been given dietary advice previously, $75.6 \%$ reported finding it either very easy or easy to incorporate personal dietary advice from their healthcare team into SW's healthy eating plan and this did not differ significantly dependent on type of diabetes (T1D 68.4\%; T2D 76.5\%, p<0.05). $14.6 \%(n=32)$ found it neither easy nor difficult to incorporate their previous healthcare practitioners dietary advice. 9.2\% $(n=20)$ respondents found some level of difficulty.

The free-text responses as to why these 20 respondents had found some level of difficulty when trying to combine the dietary guidance provided by SW to that provided by their healthcare teams was coded and thematically analysed and interpreted alongside quantitative data. Two main themes emerged:

'carbohydrate intake' and 'other'.

\section{'Carbohydrate intake'}

Whilst $108(42.1 \%)$ of total participants had been given some advice by their healthcare team to reduce carbohydrate intake with $38.3 \%$ restricting starchy foods, just some of the 20 free-text respondents reported the carbohydrate intake when following SW's food optimising plan was too generous and conflicted with 
some of the advice they had previously been given.

'Food Optimising does not fit with the previous high fat, low carb advice I have been given' 'the advice seems fruit-centred and too high in carbs'

\section{'the portions of potatoes, rice and pasta are too generous'}

'the advice I have previously been given about bread, is different'

The second theme 'other' included some limited but diverse comments as to why these respondents had found the SW dietary advise quite difficult;

\section{'i crave sweet things'}

'I am vegetarian and finding it difficult to eat sufficient protein' 'I am poor at planning meals'

For one respondent the restrictions on Healthy Extras and high energy-dense, nutrient-poor foods meant that they did not have sufficient carbohydrate allowance to enable them to have bread for supper to prevent overnight hypos.

\section{Discussion}

This service evaluation found that SW support was effective in reducing weight and BMI in adults with T1D and T2D who had either self-referred or been recommended by a healthcare professional to attend the weekly community-based group sessions or to receive the online support. Over $80 \%$ of the respondents had T2D and nearly 90\% were female. However, people of different ages and different lengths of diabetes diagnosis accessed the support. The length of membership varied with the median length being 1-2 years but some of the respondents had accessed the support for 3-5yrs or longer - this may have been continuous or noncontinuous. At the time of the survey, a third of the respondents had achieved a weight loss of $15 \mathrm{~kg}$ or more and over $60 \%$ had achieved a $10 \%$ weight loss or greater. Glycaemic control significantly improved in those respondents for whom HbA1c levels were available. Over $85 \%$ reported a reduction in HbA1c levels with a mean reduction of $21.9 \mathrm{mmol} / \mathrm{mol}$ and this increased to 27.9 $\mathrm{mmol} / \mathrm{mol}$ in those achieving $\geq 10 \%$ weight loss at the time of the survey. A positive correlation was observed between reductions in HbA1c levels and weight loss with those achieving a weight loss of $\geq 10 \%$, over 7 times more likely to achieve a HbA1c below $48 \mathrm{mmol} /$ mol. Over half of respondents reporting this level of weight loss also suggested that their diabetes diagnosis had been reversed. Changes in HbA1c were not influenced by age or length of diagnosis. Whilst more than $40 \%$ of the respondents indicated that the healthcare team had reduced their diabetes medications as a consequence of their weight loss, this number also increased to over $60 \%$ in those losing $\geq 10 \%$.

Despite the improved glycaemic control, hypoglycaemia was not that frequent in the majority and whilst some people did find it difficult to manage hypoglycaemia whilst trying to manage their weight, a similar number found it easy to. Besides making dietary changes, over three-quarters of people taking part in the survey indicated that they had increased their physical activity levels and around a half had also seen improvements in other health conditions such as raised cholesterol levels, hypertension, joint pain and anxiety. Mental-health related quality of life improved considerably and was directly related to the weight loss experienced. The demographics and findings are similar to that reported in a previous survey-based study [11] where joining BMI was $38.9 \pm 7.83$ vs. $38.7 \pm 7.5$ although mean weight loss at the time of this survey was slightly higher (- $13.0 \pm 9.03 \%$ vs. $-10.0 \pm 8.0 \%)$. The improvement in glycaemic control is also consistent with published evidence regarding SW's efficacy in this population [11,12].

Weight loss appears to be the dominant determinant of improvements in glycaemic control as measured by HbA1c levels and is in agreement with a recently published narrative review looking at the efficacy of different dietary approached to diabetes management [5]. Findings of the present study are in agreement with a systematic review and meta-analysis that reported that at least $5 \%$ weight loss was required to achieve significant reductions in HbA1c in people with T2D [13]. The Look AHEAD trial, which used an intensive intervention with a low calorie diet and an intensive physical activity programme and regular healthcare counselling, reported a $-7.8 \%$ weight loss and $-0.7 \% \mathrm{HbA} 1 \mathrm{c}$ reduction at one year while the DIRECT study reported a 9.9\% mean weight loss and a $-0.9 \%$ mean reduction in $\mathrm{HbA1c}$ at one year follow-up using a low calorie total diet replacement $[14,15]$. In comparison, the less restrictive and more 'normal' diet, with community-based or online support, followed by participants in this service evaluation achieved a greater weight loss and a reduction of $2.3 \%$ in $\mathrm{HbA} 1 \mathrm{c}$ levels in people with T2D.

In the present study, greater weight losses $(\geq 10 \%$ of initial weight) resulted in greater improvements in glycaemic control and a higher probability of achieving a glycaemic target of $48 \mathrm{mmol} / \mathrm{mol}$ or less, which is consistent with the findings of a meta-analysis by Gummesson, Nyman, Knutsson et al, [16] who reported a positive correlation between weight and HbA1c reductions in T2D. Their model estimated that for each one kilogramme lost, there would be $0.1 \%$ reduction in $\mathrm{HbA} 1 \mathrm{c}$ although this was exceeded in this study where mean weight change was $-14.2 \mathrm{~kg}$ and mean $\mathrm{HbA} 1 \mathrm{c}$ change, $-2.3 \%$. Weight and BMI reductions reported by respondents with T1D were similar to those with T2D but, whilst not statistically different, there was a $1.2 \%$ difference in Hb1Ac change between the two groups with those with T2D achieving a greater reduction which may be expected given the different underlying pathology. Faucher, Poitou, Carette et al, [17] also reported that after bariatric surgery, people with T1D reduced their HbA1c levels by $0.7 \%$ while people with T2D achieved a mean $2.1 \%$ reduction $(\mathrm{p}=0.04$ for between-type difference) despite similar weight reductions (27.9 $\%$ vs. $26.1 \%$ ). The difference in diabetes mechanism between the two subtypes may explain this difference. Weight loss in the context of T1D improves insulin sensitivity which decreases required insulin doses and can improve glycaemic control but does not address the primary abnormality which is insufficient endogenous insulin secretion. In addition, particular challenges faced more 
frequently by people with T1D, such as hypoglycaemia prevention and management, may contribute to the difference in response between people with T1D and T2D.

Evidence regarding the impact of length of diabetes diagnosis and the ability for weight loss to be associated with reduced levels of $\mathrm{HbA1c}$, leading to reversal of T2D in some cases, generally has found that people with T2D who have been diagnosed for less than 5-6 years are likely to benefit the most [15]. However, age, length of diabetes diagnosis and use of insulin therapy did not significantly impact HbA1c changes in the present study. This may be an indication that the lifestyle changes encouraged and support received were equally effective in improving glycaemic control across different demographics and contexts although a statistical difference might have not been detected because of underpowered sample size in some cases.

The related literature indicates that initial improvements in glycaemic control may diminish with time [18]. This may be due to either a decrease in motivation and commitment to maintaining lifestyle changes leading to some weight regain or because rate of weight loss tends to slow with time due to physiological adaptations. Long-term maintenance of weight loss is a recognized concern [19]. Compliance and the ability to fit dietary changes into everyday life are important for long-term weight loss. It is also important that people do not receive conflicting advice as this can be confusing and can leave people wondering which advice to follow [20]. This service evaluation included respondents who had been accessing the group or online support for a number of years. This support may have been continuous or non-continuous but the median length of membership suggests that the respondents found the support practical and easy to access and follow, resulting in the successful weight losses, associated improvements in glycaemic control and improved mental- health related quality of life at the time of the survey. Of those respondents who had received some dietary advice from their healthcare team, over three-quarters found it very easy or easy to incorporate this advice with the support offered by SW. The few reported challenges related to carbohydrate intake as some had previously received low $\mathrm{CHO}$ advice, and other challenges that anyone may face when trying to make lifestyle changes, such as the need to better plan meals. Despite a greater effect of carbohydraterestricted diets on weight and glycaemic control in the short-term in people with T2D compared with high carbohydrate diets, there is no evidence for their superiority in the long term ( $\geq 12$ months) in diabetes management, and total energy intake remains the determining factor of weight loss and subsequent glycaemic control improvement [21-24]. Diabetes UK do not state an ideal amount of carbohydrate to maintain glycaemic control in T1D or T2D [25].

Hamdy, Mottalib, Morsi et al, [26] report that for people with diabetes, achieving a weight reduction of $7 \%$ or more of initial weight at one year was associated with better weight and glycaemic control at five years. From previous evaluations of SW efficacy, this is an achievable weight loss for people who access SW support [27]. The respondents in this study had been receiving either continuous or non-continuous support for a number of years but a previous study suggests that while people who remained engaged with the support, retained greater reductions even those who stopped accessing the support maintained some weight loss [28]. The behavioural change and skills acquired may assist weight loss maintenance. For respondents who had been members for five years or more and were able to report their HbA1c levels before joining and at the time of the survey, their mean HbA1c levels at five years were still significantly lower than at baseline $(-16.9 \pm 24.85 \mathrm{mmol} / \mathrm{mol})$. The literature suggests that a period of tight glycaemic control, even if not sustained, induces better longterm outcomes in terms of vascular complications compared with continuous poor control [29].

Current guidelines recommend providing individualised and continuous dietary and lifestyle advice by expert healthcare practitioners to people with T1D and T2D, and to support those affected by overweight or obesity to reduce their weight [30, $31,25]$. However, data from the present study indicates that people with diabetes who get specific guidance by their primary healthcare practitioner regarding weight loss strategies and those referred to a weight management intervention are a minority. This is consistent with the substantial gap reported in Canada between the proportion of patients that physicians think would benefit from dietary counselling and the rate of referrals [32]. There are limited healthcare resources in both primary and secondary care and high numbers of people with diabetes and obesity who would benefit from support. Whilst some practitioners might be reluctant to refer to commercial programmes, referral to such programmes has proved to be more effective than standard healthcare advice [33,34]. Referral can lead to self-funding with two out of every five people referred found to continue self-funding and accessing support after their referral period and with no differences in weight loss between those referred and those self-funding $[35,36]$. The present study suggests that referral from a primary care practitioner is associated with an additional benefit in terms of glycaemic control compared with people with diabetes who self-referred. The encouragement and powerful endorsement by a healthcare practitioner may have contributed to the greater reductions in $\mathrm{HbA1}$ c levels seen in this minority group.

\section{Limitations of the Study}

This service evaluation includes data reported at the time of the survey where the respondents may have continued to further improve their weight loss and glycaemic management or not. There is no control group. Because it is survey-based, selection bias is possible where people who are highly engaged might constitute the majority of respondents. Recruitment only took place over a short period of time and the participants may not be totally representative of all those people with diabetes who access SW support. Weight and HbA1c data were self-reported, which increases the risk of reporting errors and respondents might have been inclined to overreport their outcomes. Not all respondents were able to report their HbA1c levels. 


\section{Conclusions}

The support provided by Slimming World, whether people were referred or chose to access the support themselves, proved to be an easy to access, effective and sustainable strategy in reducing weight and improving glycaemic control in people with both T1D and T2D, leading to a reduction in diabetes medications. Other weightrelated health conditions and mental-health related quality of life improved. Weight reduction was the main driver of the improved glycaemic control with a weight loss of $\geq 10 \%$ associated with significant $\mathrm{HbA} 1 \mathrm{c}$ reduction and a high chance of reaching a HbA1c target of $\leq 48 \mathrm{mmol} / \mathrm{mol}$. Referral by a healthcare practitioner proved beneficial in further enhancing glycaemic control in the smaller group of people who had had this encouragement. The people taking part in this service evaluation had accessed the support for a number of years suggesting that the availability of ongoing support may assist in long-term weight loss maintenance and the associated improved glycaemic control.

\section{Author Contributions}

AA: survey development, data analysis and paper writing; JB: survey development and data collection; ZB: data analysis and data description; CP: survey development and paper proof reading; JT: data analysis and paper proof reading; SB \& JL: paper proof reading

\section{References}

1. Lim E, Hollingsworth K, Aribisala B, Chen M, Mathers J, Taylor R (2011) Reversal of type 2 diabetes: normalisation of beta cell function in association with decreased pancreas and liver triacylglycerol. Diabetologia 54(10): 2506-2514.

2. Ajala O, English P, Pinkney J (2013) Systematic review and meta-analysis of different dietary approaches to the management of type 2 diabetes. The American Journal of Clinical Nutrition 97(3): 505-516.

3. Booth H, Khan O, Prevost T, Reddy M, Dregan A, et al. (2014) Incidence of type 2 diabetes after bariatric surgery: population-based matched cohort study. The Lancet Diabetes \& Endocrinology 2(12): 963-968.

4. Chaudhry Z, Doshi R, Mehta A, Jacobs D, Vakil R, et al. (2016) A systematic review of commercial weight loss programmes effect on glycemic outcomes among overweight and obese adults with and without type 2 diabetes mellitus. Obesity Reviews 17(8): 758-769.

5. Brown A, McArdle P, Taplin J, Unwin D, Unwin J, et al. (2021) Dietary strategies for remission of type 2 diabetes: A narrative review. J Hum Nutr Diet 1-14.

6. Corbin K, Driscoll K, Pratley R, Smith S, Maahs D, et al. (2018) Obesity in Type 1 Diabetes: Pathophysiology, Clinical Impact, and Mechanisms. Endocrine Reviews 39(5): 629-663.

7. Mottalib A, Kasetty M, Mar J, Elseaidy T, Ashrafzadeh S (2017) Weight Management in Patients with Type 1 Diabetes and Obesity. Current Diabetes Reports 17(10): 92.

8. Kirwan J, Aminian A, Kashyap S, Burguera B, Brethauer S (2016) Bariatric Surgery in Obese Patients with Type 1 Diabetes. Diabetes Care 39(6): 941-948.

9. Centers for Disease Control and Prevention (CDC) (2020) The Health Effects Of Overweight And Obesity | Healthy Weight | CDC

10. Mukherjee N, Chaturvedi SK (2019) Depressive symptoms and disorders in type 2 diabetes mellitus. Curr Opin Psychiatry 32(5): 416-421.
11. Avery A, Nagar R, Hillier S, Pallister C (2017) Impact on weight and glycaemic control in adults with diabetes attending a group- based commercial weight management programme. Practice Nursing 28 (2): 60-66.

12. Avery A, Griffin J, Stokes J, Coulton R, Pallister C, et al. (2019) The benefits of non-surgical weight management on weight and glycaemic control in people with complex type 2 diabetes: A primary care service evaluation of clinical outcomes at 12 months. Endocrinology, Diabetes \& Metabolism 2(2): e00045.

13. Franz M, Boucher J, Rutten-Ramos S, VanWormer J (2015) Lifestyle Weight-Loss Intervention Outcomes in Overweight and Obese Adults with Type 2 Diabetes: A Systematic Review And Meta-Analysis of Randomized Clinical Trials. J Acad Nutr Diet 115(9): 1447-1463.

14. Pi-Sunyer X, Blackburn G, Brancati F, Bray G, Bright R, et al. (2007) Reduction in Weight and Cardiovascular Disease Risk Factors in Individuals with Type 2 Diabetes: One-year results of the Look AHEAD trial. Diabetes Care 30(6): 1374-1383.

15. Lean ME, Leslie WS, Barnes AC, Brosnahan N, Thom G, et al. (2018) Primary care-led weight management for remission of type 2 diabetes (DiRECT): An open-label, cluster-randomised trial. Lancet 391(10120): 541-551.

16. Gummesson A, Nyman E, Knutsson M, Karpefors M (2017) Effect of weight reduction on glycated haemoglobin in weight loss trials in patients with type 2 diabetes. Diabetes, Obesity and Metabolism 19(9): 1295-1305.

17. Faucher P, Poitou C, Carette C, Tezenas du Montcel S, Barsamian C, et al. (2016) Bariatric Surgery in Obese Patients with Type 1 Diabetes: Effects on Weight Loss and Metabolic Control. Obesity Surgery 26(10): 23702378.

18. Swinburn B, Metcalf P, Ley S, (2001) Long-Term (5-Year) Effects of a Reduced-Fat Diet Intervention in Individuals with Glucose Intolerance. Diabetes Care 24(4): 619-624.

19. Anderson J, Konz E, Frederich R, Wood C (2001) Long-term weight-loss maintenance: A meta- analysis of US studies. The American Journal of Clinical Nutrition 74(5): 579-584.

20. Avery A (2021) Is E-Learning the Way Forward to Ensure the Provision of Accurate, Consistent and Up- To-Date Nutrition-Related Messages Across Population Groups? Komp Nutr Diet 1(3): 97-99.

21. van Wyk H, Davis R, Davies J (2015) A critical review of low-carbohydrate diets in people with Type 2 diabetes. Diabetic Medicine 33(2): 148-157.

22. Snorgaard O, Poulsen G, Andersen H, Astrup A (2017) Systematic review and meta-analysis of dietary carbohydrate restriction in patients with type 2 diabetes. BMJ Open Diabetes Research \& Care 5(1): e000354.

23. Sainsbury E, Kizirian N, Partridge S, Gill T, Colagiuri S, et al. (2018) Effect of dietary carbohydrate restriction on glycemic control in adults with diabetes: A systematic review and meta-analysis. Diabetes Research and Clinical Practice 139: 239-252.

24. McArdle P, Greenfield S, Rilstone S, Narendran P, Haque M, et al. (2019) Carbohydrate restriction for glycaemic control in Type 2 diabetes: A systematic review and meta-analysis. Diabetic Medicine 36(3): 335-348.

25. PA Dyson, D Twenefour, C Breen, A Duncan, E Elvin, et al. (2018) Diabetes UK evidence-Based Nutrition Guidelines for the Prevention and Management of Diabetes. Diabet Med 35(5): 541-547.

26. Hamdy O, Mottalib A, Morsi A, El-Sayed N, Goebel-Fabbri, et al. (2017) Long-term effect of intensive lifestyle intervention on cardiovascular risk factors in patients with diabetes in real-world clinical practice: A 5-year longitudinal study. BMJ Open Diabetes Research \& Care 5(1): e000259.

27. Stubbs RJ, Morris L, Pallister C, Horgan G, Lavin JH (2015) Weight outcomes audit in 1.3 million adults during their first 3 months' 
attendance in a commercial weight management programme. BMC Public Health 15: 882.

28. Avery A, Toon J, Kent J, Holloway L, Lavin J, et al. (2021) Impact of COVID-19 on health- related behaviours, well-being and weight management. BMC Public Health 21(1): 1152.

29. Chatterjee S, Davies M, Khunti K (2017) How to Improve Long-Term Glycemic Control in the Real World - Medicographia.

30. National Institute for Health and Care Excellence (2009) 1 Recommendations | Type 2 Diabetes In Adults: Management | Guidance | NICE.

31. National Institute for Health and Care Excellence (2015) Overview | Type 1 Diabetes In Adults: Diagnosis And Management | Guidance | NICE.

32. Wynn K, Trudeau JD, Taunton K, Gowans M, Scott I (2010) Nutrition in primary care : Current practices, attitudes, and barriers. Canadian Family Physician 56 (3): 109-116

33. Jebb S, Ahern A, Olson A, Aston L, Holzapfel C, et al. (2011) Primary care referral to a commercial provider for weight loss treatment versus standard care: A randomised controlled trial. The Lancet 378(9801): 1485-1492.

34. Aveyard P, Lewis A, Tearne S, Hood K, Christian-Brown A, et al. (2016). Screening and brief intervention for obesity in primary care: A parallel, two-arm, randomised trial. The Lancet 388: 2492.

35. Binks M, O Neil P (2002) Referral sources to a weight management program. Journal of General Internal Medicine 17(8): 596-603.

36. Stubbs J, Brogelli D, Allan J, Pallister C, Whybrow S, et al. (2013) Service evaluation of weight outcomes as a function of initial BMI in 34,271 adults referred to a primary care/commercial weight management partnership scheme. BMC Research Notes 6: 161.

\section{(c) (?) \\ This work is licensed under Creative Commons Attribution 4.0 License}

To Submit Your Article Click Here:

Submit Article

DOI: 10.32474/AD0.2021.03.000172

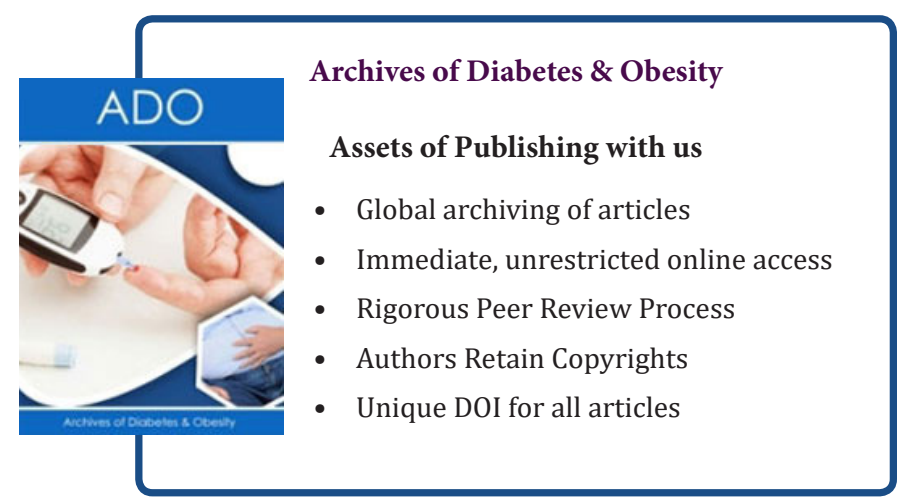

